\title{
Best from Waste: Therapeutic Potential of Plant Waste (Seeds, Peels, Flowers)
}

\author{
Savan Donga* and Sumitra Chanda
}

\author{
Phytochemical, Pharmacological and Microbiological Laboratory, \\ Department of Biosciences (UGC-CAS), Saurashtra University, \\ Rajkot-360 005, Gujarat, India \\ *Corresponding author
}

\section{A B S T R A C T}

\section{Keywords}

Seeds, Peels, Flowers, Bioactive compounds, Medicinal plants, Plant waste, Nanoparticles, Biological activity

Article Info

Accepted:

22 July 2020

Available Online:

10 August 2020
Bio waste which includes fruit and vegetable peels, seeds, pomace, rind are generated in enormous amounts and discarded into the environment adding to pollution. Flowers which are generally used for decoration and religious purpose are also thrown into nature as unwanted material. They create lot of waste and are also hazardous. However, these parts of the plant are seeds, endowed with phytoconstituents and sometimes more than those present in other parts. The best was to minimize this hazard is making use of them in food, pharmaceutical and allied industries after proper extraction of bioactive compounds from them. To enumerate this idea, in this review, we have enlisted seeds, peels and flowers of 60 different plants along with their biological activity and bioactive compounds present in them. Some are used directly as crude extracts while some are used to synthesize nanoparticles which show promising biological activities. Thus, plant waste i.e. seeds, peels and flowers can be used profitably as a source of natural medicine or ingredients in many industries. Some activities are reported but other activities can be tried out. Detailed structurally analysis also should be done which may give new lead molecules or new drugs to be used as safe, natural and novel antimicrobics or antioxidants or anticancer or antiulcer agents. This review undeniably and definitely opens up the possibility for utilization of these plant waste products for therapeutic and industrial purpose.

\section{Introduction}

Medicinal plants are important sources for discovering new drugs for many diseases and disorders. From times immemorial, plants are being used to cure many ailments and recently the trend of use of this green medicine has increased. This is merely because medicinal plants are free from many side effects that are generally associated with synthetic drugs, they are easily available and affordable by all the people. The diversity of medicinal plants is vast and there is cure for any and every ailment in them. They may be directly used as drugs or they may act as lead molecules for the discovery of new drug candidates. Many of the drugs used for deadly diseases like cancer are of plant origin (Chanda and Nagani, 2013). 
Plants show various biological activities viz. antioxidant (Punica granatum - Kaneria et al., 2012), hepatoprotective (Abelmoschus moschatus - Singh et al., 2012), anticancer and antimicrobial and synergistic antimicrobial (Pterocarpus santalinus Donga et al., 2017a, 2017b), antioxidant and anti-inflammatory (Moringa oleifera - Xu et al., 2019), anti-ulcer (Nigella sativa - Paseban et al., 2020), antiurolithiatic (Mangifera indica - Iman et al., 2020) etc. The therapeutic property is not isolated to any specific part of the plant. All plant parts show medicinal properties for eg. fruit and vegetable peels showed antimicrobial activity (Rakholiya et al., 2014), Emblica officinalis fruit showed anti-inflammatory activity (Golechha et al., 2014), Mangifera indica stem bark showed anti-viral activity (AbdelMageed et al., 2014), Nephelium lappaceum peels showed antidiabetic activity (Ma et al., 2017), aerial parts of Polygonum equisetiforme showed hepatoprotective property (El-Toumy et al., 2019), Pouteria caimito peel showed antimicrobial and antidiarrheal activity (Abreu et al., 2019), Opuntia ficus indica seed oil showed protection against gastric ulcer (Khemiri and Bitri, 2019); Lawsonia inermis and Murraya koenigii seed extract also showed antiulcer activity (Eggadi et al., 2019). Lavendula bipinnata leaves showed antioxidant activity (Pande and Chanda, 2020) while Annona squamosa leaf showed anticancer effect (AlNemari et al., 2020), Carica papaya flowers showed antioxidant and antibacterial activity (Dwivedi et al., 2020), Beta vulgaris root showed antimicrobial and anticancer activities (El-Mesallamy et al., 2020), etc.

Plant parts are enriched with phytoconstituents like alkaloids, flavonoids, phenols, tannins, saponins, glycosides, steroids, etc. But their concentration varies from part to part and hence the therapeutic efficacy of plant part also varies. The leaf of the plant may show very good antioxidant activity but stem or seed may not show similar activity. The phytoconstituents may act individually or synergistically. Plant secondary metabolites are bioactive molecules that are not essential for plant survival, but have important role in plant growth, development, reproduction and protection from predators and environmental stresses.

Fruits and vegetables generate lot of biowaste in the form of peels and seeds which are thrown into the environment. They increase pollution and their discard is a major problem. Flowers are another part of the plant which are generally used for decoration or religious purpose. They are also discarded into the environment increasing biowaste. However, these parts can be used as a source of natural antioxidant, antimicrobial or antiulcer or antidiabetic agent. They are also rich in various phytoconstituents like any other part of the plants. The therapeutic use of these parts will reduce environmental pollution and this is the best use of the waste. The discarded peels, seeds or flowers can be used as gelling and thickening agents in the refined foods; Polysaccharides from them can be a source of gum and can be used as thickeners, gelling agents, texture modifiers and stabilizers; as a source of bio-pigments like carotenoids and colourants, as emulsifiers, essential oils, organic acids and minerals, as substrate for microbial fermentation for enzymes production, for bio-ethanol/methanol production, for production of biodegradable plastics, as bio fuels and biofertilizers, biopesticides, bio-preservatives, mushroom cultivation, etc (Wadhwa et al., 2015).

There are many types of seeds. Some seeds are used as spices (Cuminum cyminum, Trigonella foenum-graecum, Coriandrum sativum, Nigella sativa, Foeniculum vulgare), some seeds are eaten along with fruits and 
vegetables (Solanum lycopersicum, Pisum sativum, Cicer arietinum, Psidium guajava, Actinidia deliciosa) while some are thrown into the environment (Carica papaya, Cucumis melo, Manilkara zapota, Citrus limon, Momordica dioica). However, seeds possess various phytoconstituents and can be therapeutically used. The seeds may have extractible high value-added components. Seeds showed various biological activities like antioxidant, anti-inflammatory, antimicrobial, antidiabetic, antidiarrheal, wound healing, etc (Table 1). All these properties are because of the phytoconstituents present in them in different concentrations which act individually or synergistically. Mesua ferrea seed extract showed antimicrobial activity (Chanda et al., 2013). Mangifera indica seed kernel showed inhibition of Pseudomonas spp (Rakholiya et al., 2015). Oil extracted from seeds of Citrus sinensis (orange) showed antioxidant activity; they contained phenols, carotenoids, phytosterols and $\alpha$-tocopherols (Jorge et al., 2016). Parikh and Patel (2017) reported antioxidant activity by Manilkara hexandra fruit and seeds; the fruits contained phenolics like gallic acid, quercetin and kaempferol, while seeds contained quercetin, gallic acid and vanillic acid. 11 varieties of Phoenix dactylifera (date palm) seeds were evaluated for phenol, flavonoid and anthocyanin content and antibacterial and antioxidant properties by Metoui et al., (2019) and reported a direct correlation between phenolic content and inhibitory activity. Cucumis melo (melon) seeds and peels showed antioxidant and anticancer activities (Rolim et al., 2018). The seeds and peels contained phenols, flavonoids and tannins; they also reported was a direct correlation between phytochemical content and antioxidant and anticancer activities. Eriobotrya japonica (loquat) seed starch showed antioxidant activity (Barbi et al., 2018). The seeds from unripe fruit had higher polyphenol content and higher antioxidant activity. Myrciaria dubia (Camu-camu) seed coat showed antioxidant and antihypertensive activity (Fidelisa et al., 2018). They were rich in phenolic acids and flavonoids. The aqueous extract showed higher antioxidant activity; it contained total phenolics, non-tannin phenolics, (-)-epicatechin, chlorogenic acid, 2,4-dihydroxybenzoic acid, 2,5dihydroxybenzoic acid and gallic acid. On the other hand the propanone extract showed higher antihypertensive activity and $\mathrm{Cu}^{2+}$ chelating ability; it had higher levels of quercetin, quercetin-3-rutinoside (rutin), tresveratrol, ellagic, caffeic, rosmarinic, ferulic, and p-coumaric acids. The ethanolic extract possessed only condensed tannins, syringic acid, and (-)-epicatechin. The extracting solvent plays an important role in extracting the phytoconstituents or bioactive compounds from this biowaste and in exhibiting a particular activity. Parkia speciosa seeds possessed phenols, flavonoids, terpenoids and alkaloids and showed antimicrobial and antioxidant activities (Ghasemzadeh et al., 2018). There was a significant correlation between biological activity and flavonoid content followed by phenolic content. Durio zibethinus (durian) seeds showed antimicrobial, cytotoxic and photocatalytic activity (Sumitha et al., 2018).

The pulp, peel and seed of four avocado varieties (Persea americana) were investigated for their antibacterial and antioxidant activities (Amado et al., 2019). The peels and seeds showed more antioxidant activity because they possessed more antioxidant compounds, phenols and flavonoids. Similar results were found in peels and seeds of Hass and Fuerte avocado varieties (Rodriguez-Carpena et al., 2011). The antibacterial activity was more in peels followed by seeds. Datura stramonium seed showed anti-inflammatory activity in carrageenan induced paw edema model in Wistar albino rats (Agarwal et al., 2019). 
Moringa oleifera leaves, seeds and roots showed antioxidant and anti-inflammatory activities ( $\mathrm{Xu}$ et al., 2019); all the parts were rich in flavonoids and phenolic acids. There was a direct correlation between phytochemical content and observed activities. Pouteria campechiana seed polysaccharides ultrasonic-assisted extracted showed antioxidant activity (Ma et al., 2019). Wound healing activity was shown by seeds of Chrysophyllum Albidum (Babatunde et al., 2019). Garcinia mangostana (mangosteen) peel and seeds are waste products that can be recycled into medical and pharmaceutical applications due to their structures and properties. They have antibiotic properties and hence are suitable as bio-fillers in natural rubber products like medical gloves, rubber transdermal patches, rubber toys, etc (Moopayak and Tangboriboon, 2020). Garcinia kola and Buchholzia coriacea seeds (Abubakar et al., 2020) showed antioxidant activity; $G$. kola and B. coriacea seeds contained phenols, flavonoids, alkaloids, saponins and tannins.

Recently, various metal nanoparticles are being synthesized from seed extracts which showed many biological activities. Silver nanoparticles synthesized using seed extract of Trigonella foenum-graecum showed anticancer activity (Varghese et al., 2019) while seed extract of Pedalium murex showed antimicrobial activity (Ishwarya et al., 2017). Elettaria cardamomum seed extract mediated synthesized gold nanoparticles showed antibacterial, anticancer and antioxidant activities (Rajan et al., 2017). Zinc nanoparticles synthesized using Elettaria cardamomum seed extract showed anticancer activity (Abbasi et al., 2019)

Fruit and vegetable peels are considered as one of the most waste products of food industry. They are generated in huge amounts and discarded into the environment increasing pollution. However, they show many medicinal properties. They can be utilized for the production of value added by - products. Peels contain many important phytoconstituents which can be used for pharmacological or pharmaceutical purposes. Researchers extracted numerous components having antimicrobial, antioxidant, antidiabetic, anticancer, hepatoprotetive, antiobesity and anti- inflammatory activities from different peels (Table 1). Actinidia deliciosa (Kiwi) peels showed antibacterial. antihelicobacter pylori and cytotoxic activity (Motohashi et al., 2001). Cucurbita moschata (pumpkin) fruit peel showed antioxidant, antibacterial and wound healing properties (Bahramsoltani et al., 2017). Antioxidant and anti-salmonella activities of eggplant peel was reported by Rochin-Medina et al., (2019). Anticancer and antibacterial properties of Citrus reticulate peels were reported by (Selim et al., 2019); they contained phenols, flavonoids and coumarone compounds. Mangifera indica peels showed antibacterial, anti-inflammatory, anti-cancer and antioxidant activities (Huang et al., 2018). The bioactive compounds in the peels were polyphenols which were responsible for the observed activities. Combination of peel extracts of Allium sativum and Allium cepa showed antidiabetic effect (Lolok et al., 2019).

Punica granatum (pomegranate) peels showed antibacterial activity against Cronobacter sakazakii (Yemis et al., 2019). The peels were rich in polyphenolic compounds especially hydrolysable polyphenolics like elligitannins $\alpha$ - and $\beta$ punicalagin followed by ellagic acid, ellagic acid derivatives and punicalin. Antimicrobial activity of $P$. granatum fruit peels was also reported by Al-Zoreky (2017). Antiinflammatory activity was reported from peels of Citrus sinensis (Osarumwense, 2017), Citrus grandis (Zhao et al., 2019), Punica 
granatum (Mastrogiovanni et al., 2019) and Ziziphus jujuba (Wang et al., 2019). Citrus grandis peels were rich in coumarins; Punica granatum peels were rich in high molecular weight phenols like alpha and beta punicalagin and low molecular weight phenols like gallic acid, ellagic acid and granatin $\mathrm{B}$; Ziziphus jujuba contained phenolics like quercetin galangin and flavonoids. All these secondary metabolites were responsible for the observed antiinflammatory activity. Nephelium lappaceum (Rambutan) peel extract rich in polyphenolic content showed antidiabetic activity (Ma et al. 2017); antioxidant and antidiabetic activity of Aloe vera peel extract was reported by Christijanti et al., (2019); antimicrobial activity of fruit peel extract of Pouteria caimito was reported by Abreu et al., (2019). The genus Pouteria were rich in triterpenes and flavonoids; Actinidia chinensis (Kiwi) peels showed antioxidant, antimicrobial and anticancer activity; they contained polyphenols (catachin, quercetin and epigallocatechin) and flavonoids (Alim et al., 2019).

Banana peels showed antimicrobial and antioxidant activities (Mokbel and Hashinaga 2005; Chueh et al., 2019) which may be due to the bioactive compounds present in peels. The peel had abundant phenolic content, including flavan-3-ols and flavonols ( $\mathrm{Vu}$ et $a l ., 2018$ ) and dried peel powder had catechin, epicatechin, gallocatechin and procyanidin (Rebello et al., 2014). Antioxidant and antitumor activities of Nendran banana peels rich in phenol, flavonoid and caretonoid content was reported by Kumar et al., (2019). Litchi chinensis (Lychee) peel powder showed hepatoprotetive and anti-obesity property (Queiroz et al., 2018); peels contained polyphenols, flavonoids and anthocyanins. Citrus sinensis (orange), Citrus limonia (yellow lemon) and Musa acuminate (banana) peels showed remarkable antimicrobial activity against a panel of microorganisms (Saleem and Saeed, 2020). The peels were rich in trace elements zinc, magnesium and polyphenolic content.

Metal nanoparticles of silver, gold and zinc synthesized using peel extracts also showed various biological activities. For e.g. Prunus persica peel mediated synthesized silver nanoparticles showed antioxidant activity (Patra et al., 2016). Antibacterial and antioxidant activities were reported by gold and zinc nanoparticles synthesized using Citrullus lanatus and Punica granatum peel respectively (Patra et al., 2015; Sukri et al., 2019)

Flowers have cosmetic or phytotherapeutical use; essential oils from flowers like lavender, orange blossom, jasminum and rose are used in aromatherapy and perfumes due to their soothing and calming effects. Flowers show a number of properties like antifungal, antibacterial, antioxidant, antimicrobial, antiulcer, anti-diabetic, hepatoprotective, neuroprotective, anti-cancer, antiinflammatory, etc (Table 1). Woodfordia fruticoza flowers showed protective effect against acetaminophen induced hepatic toxicity in rats (Baravalia and Chanda, 2011). Acacia saligna flowers showed antifungal, antibacterial and antioxidant activity (AlHuqail et al., 2019). The flowers contained phenols and flavonoids like benzoic acid, caffeine and o-coumaric acid, naringenin, quercetin and kaempferol. Agastache rugosa flowers showed antioxidant and antimicrobial activities and these activities were attributed to bioactive molecules present in the flowers which include terpenoids, carotenoids, and phenylpropanoids (Park et al., 2019). Oil extracted from Etlingera elatior flowers using subcritical carbon dioxide showed antibacterial activity (Anzian et al., 2020). The major chemical compounds present were polyphenols, flavonoids, anthocyanins and 
tannins. Flower extracts of Vernonia amygdalina showed antibacterial and antioxidant activity (Habtamu and Melaku, 2018); the flowers had two natural antioxidants, isorhamnetin and luteolin, which were responsible for the observed antioxidant and antibacterial activities.

\section{Graphical abstract}

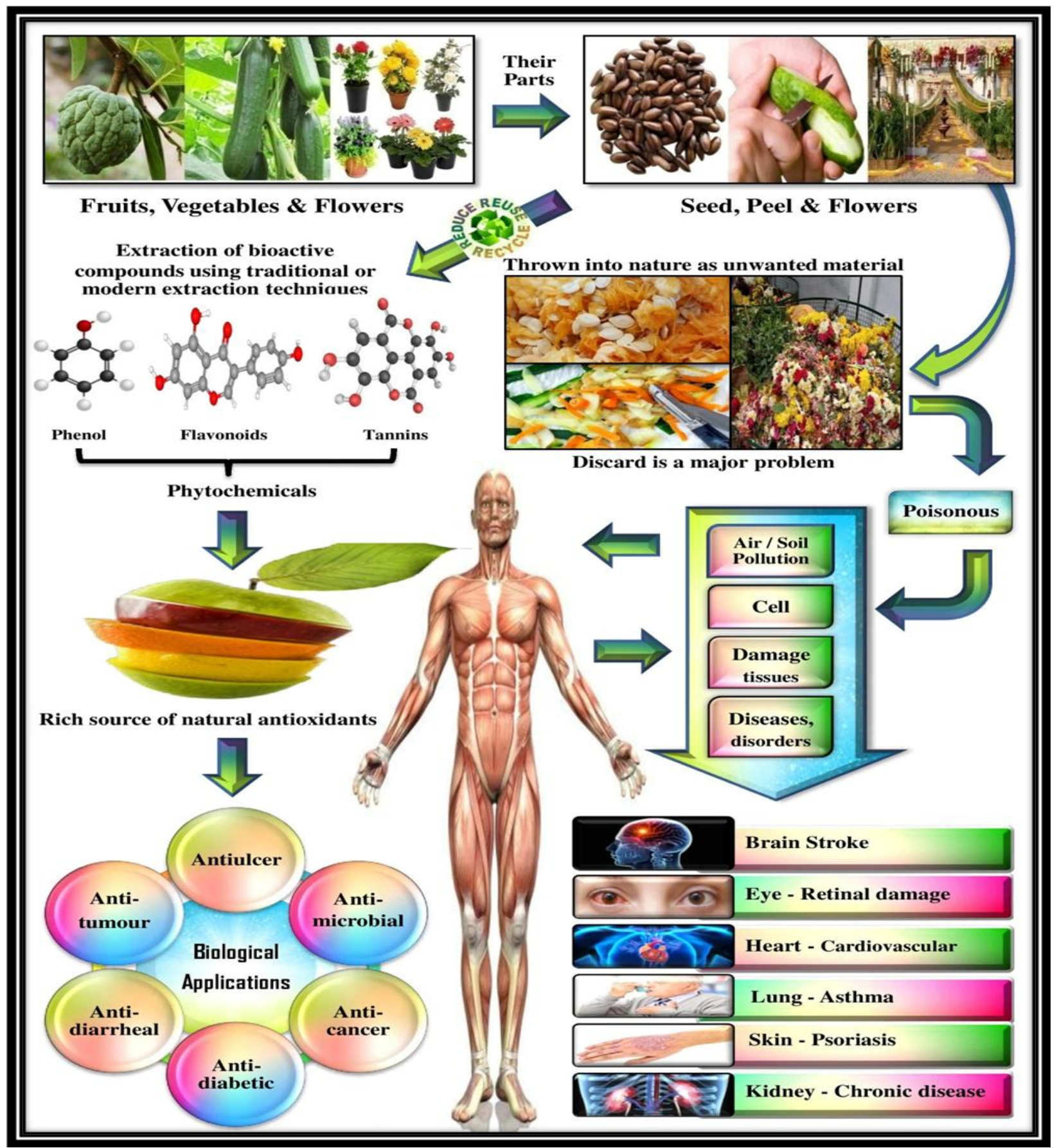


Table.1 List of plant waste i.e. seeds, peels and flowers, their family, solvent and assay used for different biological activities






\begin{tabular}{|c|c|c|c|c|c|}
\hline 6 & $\begin{array}{l}\text { Durio zibethinus L./ } \\
\text { Malvaceae }\end{array}$ & $\mathrm{AQ}$ & $\begin{array}{c}\text { SEM, } \\
\text { SAED, } \\
\text { EDX, } \\
\text { XRD, } \\
\text { DLS, } \\
\text { Zeta, } \\
\text { MIC, } \\
\text { MBC, } \\
\text { Agar disc } \\
\text { diffusion, } \\
\text { BSCA }\end{array}$ & $\begin{array}{l}\text { Antimicrobial, } \\
\text { Cytotoxic, } \\
\text { Photocatalytic }\end{array}$ & $\begin{array}{l}\text { Sumitha et al., } \\
2018\end{array}$ \\
\hline 7 & $\begin{array}{l}\text { Elettaria cardamomum } \\
\text { (L.) Maton/Zingiberaceae }\end{array}$ & AQ & $\begin{array}{c}\text { (AuNPs) } \\
\text { DPPH, } \\
\text { NO, } \\
\text { OH, } \\
\text { Agar well } \\
\text { diffusion, } \\
\text { MTT }\end{array}$ & $\begin{array}{l}\text { Antibacterial, } \\
\text { Anticancer, } \\
\text { Antioxidant }\end{array}$ & $\begin{array}{l}\text { Rajan et al., } \\
2017\end{array}$ \\
\hline 8 & $\begin{array}{c}\text { Eriobotrya japonica } \text { L./ } \\
\text { Rosaceae }\end{array}$ & AQ & $\begin{array}{c}\text { SEM, } \\
\text { XRD, } \\
\text { TG, } \\
\text { DTG, } \\
\text { DLS, } \\
\text { TPC, } \\
\text { DPPH, } \\
\text { FRAP, } \\
\text { ABTS }\end{array}$ & Antioxidant & $\begin{array}{l}\text { Barbi et al., } \\
2018\end{array}$ \\
\hline 9 & $\begin{array}{l}\text { Garcinia kola Heckel/ } \\
\text { Clusiaceae }\end{array}$ & AQ & $\begin{array}{l}\text { TPC, } \\
\text { TFC, } \\
\text { AC, } \\
\text { SC, } \\
\text { TC, } \\
\text { DPPH, } \\
\text { FRAP }\end{array}$ & Antioxidant & $\begin{array}{l}\text { Abubakar et al., } \\
2020\end{array}$ \\
\hline
\end{tabular}




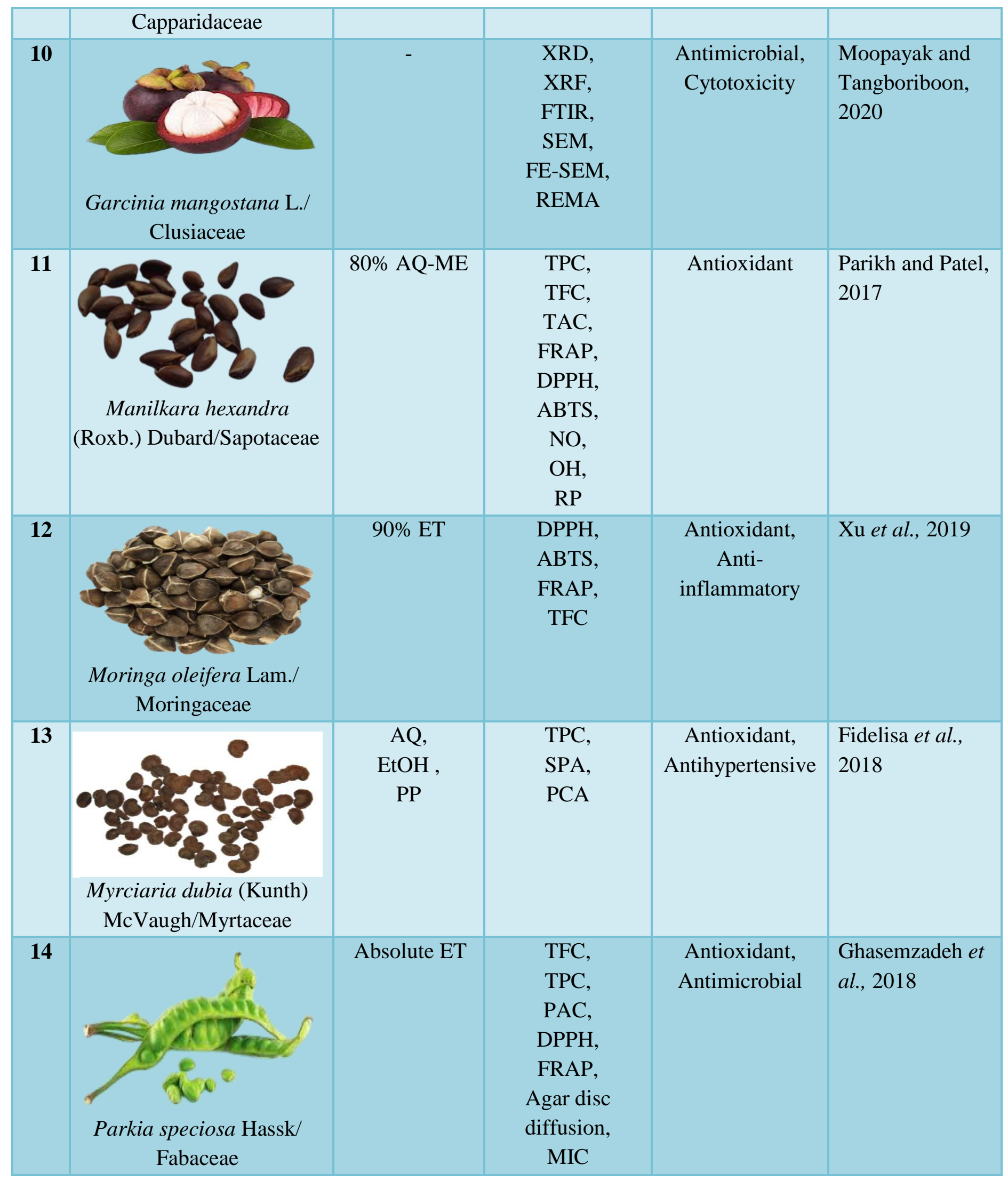




\begin{tabular}{|c|c|c|c|c|c|}
\hline 15 & $\begin{array}{l}\text { Pedalium murex L.I } \\
\text { Pedaliaceae }\end{array}$ & AQ & $\begin{array}{c}\text { (AgNPs) } \\
\text { FTIR, } \\
\text { XRD, } \\
\text { HR-TEM, } \\
\text { EDX, } \\
\text { MIC, } \\
\text { MTP, } \\
\text { EPS, } \\
\text { BVA, } \\
\text { LDCA }\end{array}$ & $\begin{array}{l}\text { Antibiofilm, } \\
\text { Antimicrobial }\end{array}$ & $\begin{array}{l}\text { Ishwarya et al., } \\
2017\end{array}$ \\
\hline 16 & $\begin{array}{c}\text { Persea americana Mill./ } \\
\text { Lauraceae }\end{array}$ & ET & $\begin{array}{c}\text { DPPH, } \\
\text { ABTS, } \\
\text { FRAP, } \\
\text { TPC, } \\
\text { TFC, } \\
\text { MIC, } \\
\text { MBC, } \\
\text { HA }\end{array}$ & $\begin{array}{l}\text { Antioxidant, } \\
\text { Antibacterial, } \\
\text { Toxicity testing }\end{array}$ & $\begin{array}{l}\text { Amado et al., } \\
2019\end{array}$ \\
\hline 17 & $\begin{array}{l}\text { Phoenix dactylifera } \mathrm{L} . \mathrm{I} \\
\text { Arecaceae }\end{array}$ & $\begin{array}{c}80 \% \mathrm{ME}, \\
85 \% \mathrm{ET}, \\
\mathrm{AQ} \\
\mathrm{ME} \\
\mathrm{AC}\end{array}$ & $\begin{array}{c}\text { TPC, } \\
\text { TFC, } \\
\text { TAC, } \\
\text { DPPH, } \\
\text { Agar disc } \\
\text { diffusion }\end{array}$ & $\begin{array}{l}\text { Antioxidant, } \\
\text { Antibacterial }\end{array}$ & $\begin{array}{l}\text { Metoui et al., } \\
2018\end{array}$ \\
\hline 18 & $\begin{array}{c}\text { Pouteria campechiana } \\
\text { (Kunth) Baehni/Sapotaceae }\end{array}$ & $\begin{array}{c}\text { PE, } \\
95 \% \mathrm{ET} \\
\text { AQ }\end{array}$ & $\begin{array}{c}\text { PSA, } \\
\text { FTIR, } \\
\text { SEM, } \\
\text { NMR, } \\
\text { DPPH, } \\
\text { SO, } \\
\text { ABTS, } \\
\text { OH }\end{array}$ & Antioxidant & Ma et al., 2019 \\
\hline 19 & $\begin{array}{l}\text { Silybum marianum (L.) } \\
\text { Gaernt./Asteraceae }\end{array}$ & AQ & $\begin{array}{c}\text { (ZnONPs) } \\
\text { MTT, } \\
\text { Agar disc } \\
\text { diffusion, } \\
\text { TAC, } \\
\text { TRP, } \\
\text { DPPH, }\end{array}$ & $\begin{array}{l}\text { Antibacterial, } \\
\text { Antioxidant, } \\
\text { Cytotoxicity }\end{array}$ & $\begin{array}{l}\text { Abbasi et al., } \\
2019\end{array}$ \\
\hline
\end{tabular}




\begin{tabular}{|c|c|c|c|c|c|}
\hline & & & $\begin{array}{l}\alpha \mathrm{AI}, \\
\mathrm{BSL}\end{array}$ & & \\
\hline 20 & 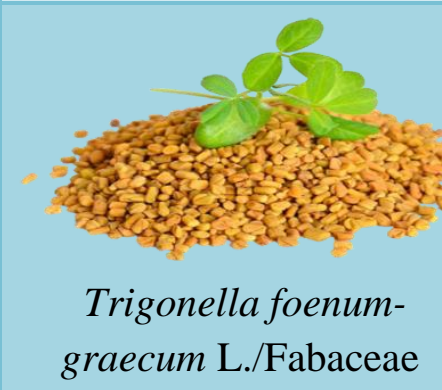 & AQ & $\begin{array}{l}\text { (AgNPs) } \\
\text { SEM, } \\
\text { XRD, } \\
\text { FTIR, } \\
\text { EDAX, } \\
\text { MIC, } \\
\text { MTT }\end{array}$ & $\begin{array}{l}\text { Antibacterial, } \\
\text { Antifungal, } \\
\text { Anticancer }\end{array}$ & $\begin{array}{l}\text { Varghese et al., } \\
2019\end{array}$ \\
\hline 21 & & AQ & $\begin{array}{l}\text { DPPH, } \\
\text { ABTS, } \\
\text { RP }\end{array}$ & $\begin{array}{l}\text { Antioxidant, } \\
\text { Metal chelation } \\
\text { activity }\end{array}$ & $\begin{array}{l}\text { Kanbargi et al., } \\
2016\end{array}$ \\
\hline & $\begin{array}{c}\text { Ziziphus jujube Mill./ } \\
\text { Rhamnaceae }\end{array}$ & & & & \\
\hline \multicolumn{6}{|c|}{ PEEL } \\
\hline 22 & $\begin{array}{c}\text { Ananas comosus (L.) } \\
\text { Merr./Bromeliaceae }\end{array}$ & $\mathrm{AQ}$ & $\begin{array}{c}\text { (ZnONPs) } \\
\text { FTIR, } \\
\text { XRD, } \\
\text { EDX, } \\
\text { FESEM, } \\
\text { TEM, } \\
\text { Agar disc } \\
\text { diffusion }\end{array}$ & Antibacterial & Basri et al., 2020 \\
\hline 23 & $\begin{array}{l}\text { Actinidia chinensis } \\
\text { Planch./Actinidiaceae }\end{array}$ & $\mathrm{ME}$ & $\begin{array}{c}\text { TPC, } \\
\text { TFC, } \\
\text { DPPH, } \\
\text { ABTS, } \\
\text { OH, } \\
\text { Agar disc } \\
\text { diffusion }\end{array}$ & $\begin{array}{l}\text { Antioxidant, } \\
\text { Antimicrobial, } \\
\text { Antiproliferative }\end{array}$ & Alim et al., 2019 \\
\hline 24 & $\begin{array}{l}\text { Actinidia deliciosa } \\
\text { (A.Chev.) C.F.Liang \& } \\
\text { A.R.Ferguson/Actinidiace }\end{array}$ & $\begin{array}{c}\mathrm{HE}, \\
\mathrm{AC}, \\
\text { Pure ME, } \\
70 \% \mathrm{ME}\end{array}$ & $\begin{array}{c}\text { MTT, } \\
\mathrm{HIV} \\
\mathrm{O}_{2}^{-}\end{array}$ & $\begin{array}{l}\text { Antibacterial, } \\
\text { Anti-helicobacter } \\
\text { pylori, } \\
\text { Cytotoxic }\end{array}$ & $\begin{array}{l}\text { Motohashi et al., } \\
2001\end{array}$ \\
\hline
\end{tabular}




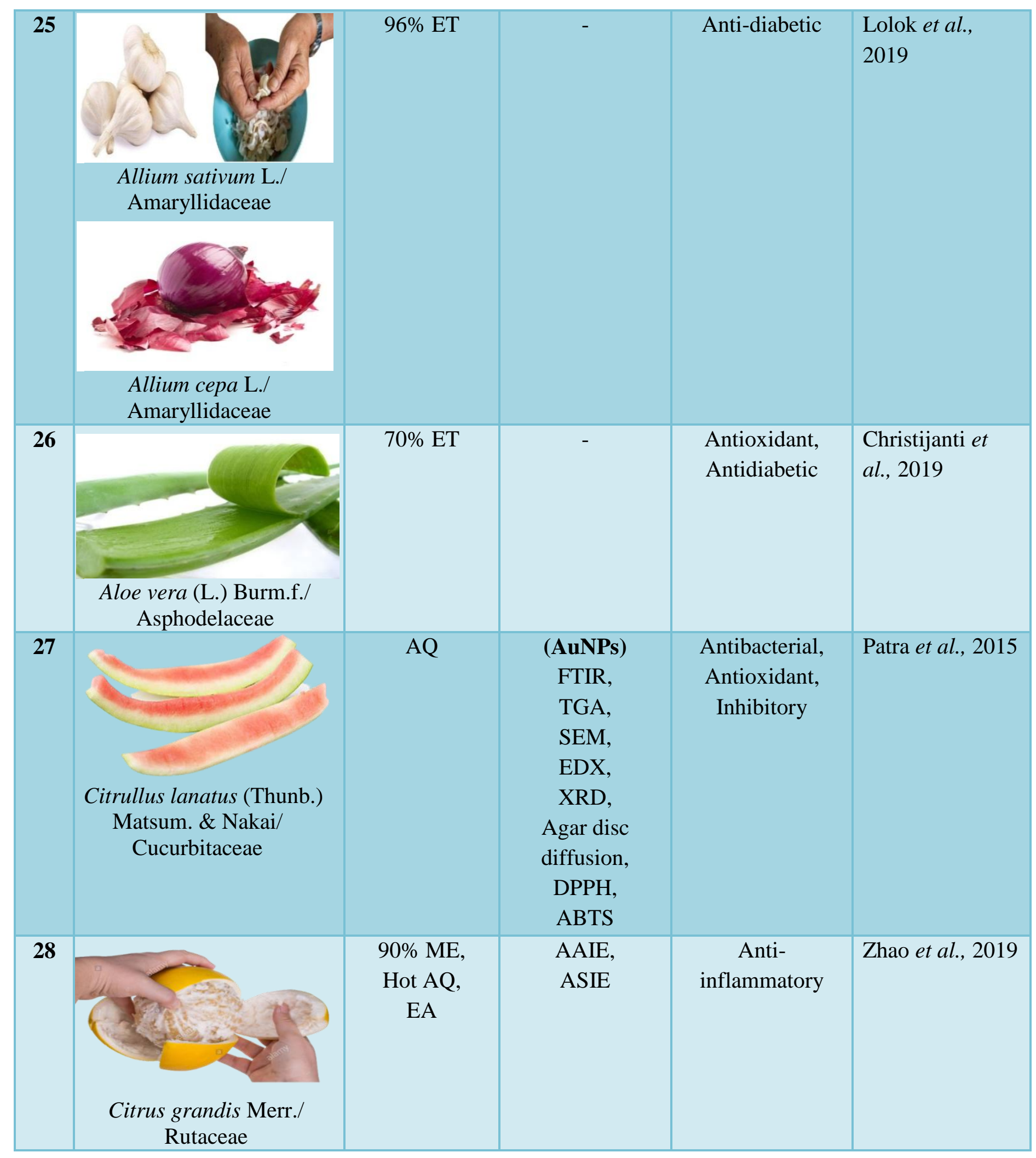




\begin{tabular}{|c|c|c|c|c|c|}
\hline 29 & $\begin{array}{c}\text { Citrus reticulata Blanco/ } \\
\text { Rutaceae }\end{array}$ & $70 \% \mathrm{ET}$ & $\begin{array}{c}\mathrm{HEPG}_{2}, \\
\mathrm{HCT}, \\
\text { MCF-7, } \\
\text { HPLC }\end{array}$ & $\begin{array}{l}\text { Anticancer, } \\
\text { Antimicrobial }\end{array}$ & $\begin{array}{l}\text { Selim et al., } \\
2019\end{array}$ \\
\hline 30 & $\begin{array}{l}\text { Citrus limon (L.) Osbeck/ } \\
\text { Rutaceae } \\
\text { Musa acuminata Colla/ } \\
\text { Musaceae }\end{array}$ & $\begin{array}{l}\text { ME, } \\
\text { EA, } \\
\text { ET, } \\
\text { AQ }\end{array}$ & $\begin{array}{c}\text { Agar well } \\
\text { diffusion, } \\
\text { MIC, } \\
\text { GC-MS }\end{array}$ & Antimicrobial & $\begin{array}{l}\text { Saleem and } \\
\text { Saeed, } 2020\end{array}$ \\
\hline 31 & $\begin{array}{c}\text { Citrus sinensis (L.) } \\
\text { Osbeck/Rutaceae }\end{array}$ & $\begin{array}{l}\text { ET, } \\
\text { ME }\end{array}$ & - & $\begin{array}{c}\text { Anti- } \\
\text { inflammatory }\end{array}$ & $\begin{array}{l}\text { Osarumwense, } \\
2017\end{array}$ \\
\hline
\end{tabular}




\begin{tabular}{|c|c|c|c|c|c|}
\hline 32 & $\begin{array}{l}\text { Cucurbita moschata } \\
\text { Duchesne ex Poir./ } \\
\text { Cucurbitaceae }\end{array}$ & $70 \%$ ET & $\begin{array}{l}\text { DPPH, } \\
\text { TAC, } \\
\text { TPC, } \\
\text { TMC, } \\
\text { MIC }\end{array}$ & $\begin{array}{l}\text { Antibacterial, } \\
\text { Antioxidant, } \\
\text { Wound healing }\end{array}$ & $\begin{array}{l}\text { Bahramsoltani et } \\
\text { al., } 2017\end{array}$ \\
\hline 33 & $\begin{array}{l}\text { Litchi chinensis Sonn/ } \\
\text { Sapindaceae }\end{array}$ & ME & $\begin{array}{l}\text { SLP, } \\
\text { LP, } \\
\text { HPA }\end{array}$ & Hepatoprotective & $\begin{array}{l}\text { Queiroz et al., } \\
2018\end{array}$ \\
\hline 3 & $\begin{array}{c}\text { Mangifera indica L./ } \\
\text { Anacardiaceae }\end{array}$ & $\begin{array}{l}\text { 95\% ET, } \\
\text { NPEE, } \\
\text { CPEE, } \\
\text { NPWE, } \\
\text { CPWE }\end{array}$ & $\begin{array}{l}\text { Agar disc } \\
\text { diffusion, } \\
\text { PPC, } \\
\text { DPPH, } \\
\text { ABTS, } \\
\text { CLC, } \\
\text { MTT }\end{array}$ & $\begin{array}{l}\text { Anti } \\
\text { inflammatory, } \\
\text { Anti-cancer, } \\
\text { Antioxidant, } \\
\text { Antibacterial }\end{array}$ & $\begin{array}{l}\text { Huang et al., } \\
2018\end{array}$ \\
\hline 35 & $\begin{array}{c}\text { Musa acuminata Colla/ } \\
\text { Musaceae }\end{array}$ & - & $\begin{array}{l}\text { TPC, } \\
\text { FCC, } \\
\text { FRAP, } \\
\text { TEAC, } \\
\text { DPPH, } \\
\text { SAEA, } \\
\text { MDA }\end{array}$ & Antioxidant & $\begin{array}{l}\text { Chueh et al., } \\
2019\end{array}$ \\
\hline 36 & $\begin{array}{c}\text { Musa acuminata Colla/ } \\
\text { Musaceae }\end{array}$ & - & $\begin{array}{l}\text { TPC, } \\
\text { TFC, } \\
\text { DPPH, } \\
\text { FRAP, } \\
\text { MTT }\end{array}$ & $\begin{array}{l}\text { Antioxidant, } \\
\text { Antitumour }\end{array}$ & $\begin{array}{l}\text { Kumar et al., } \\
2019\end{array}$ \\
\hline
\end{tabular}




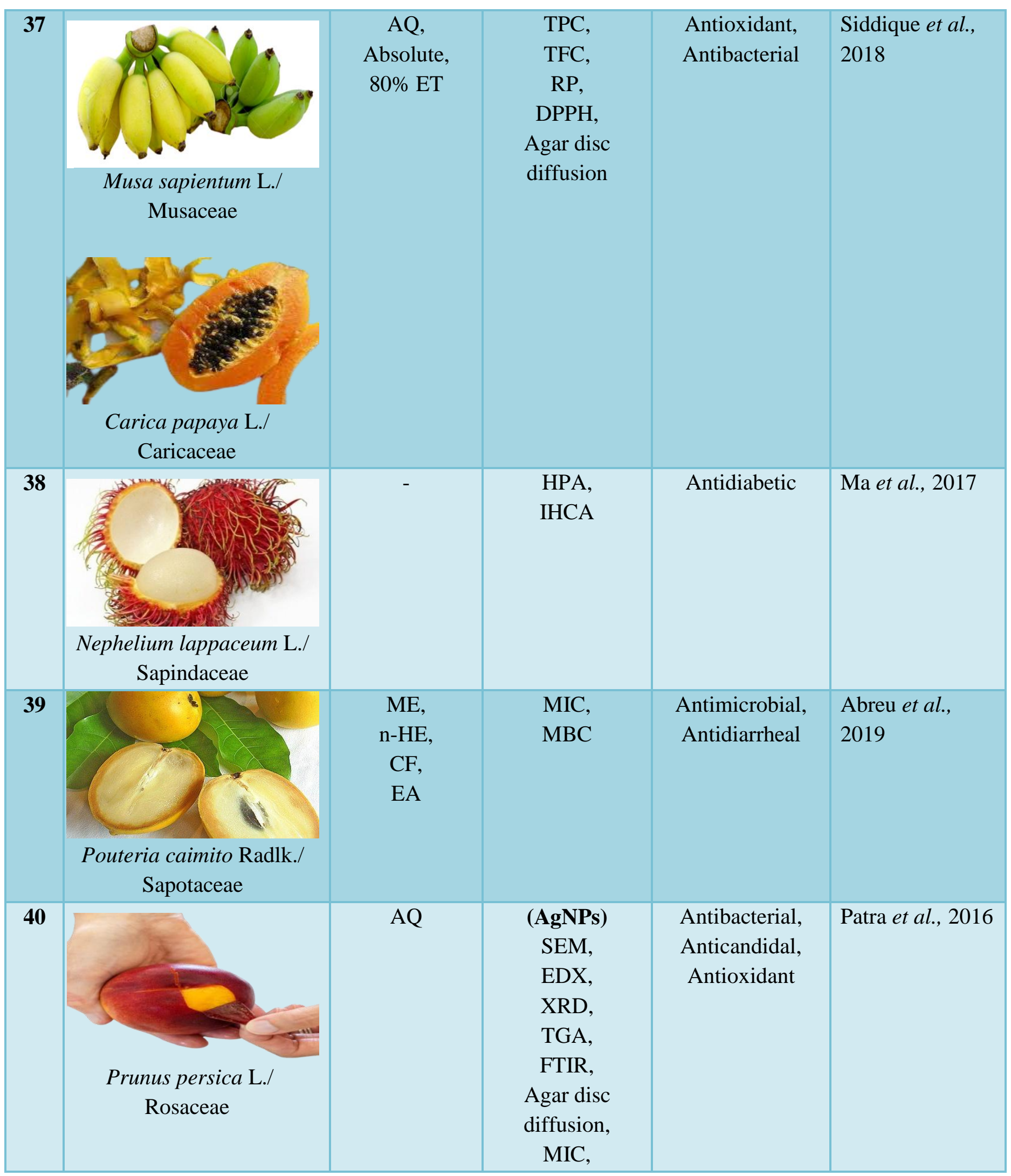




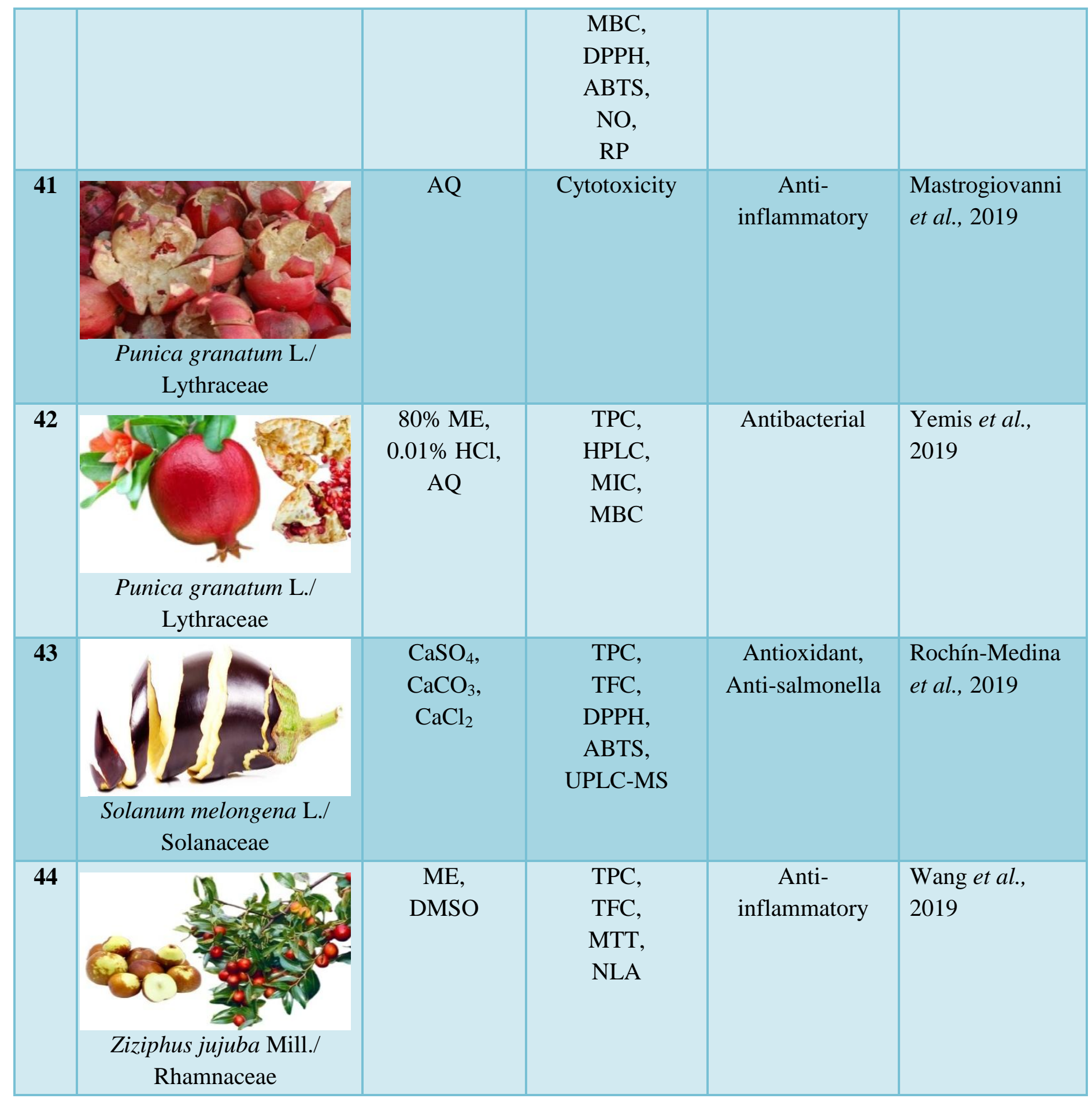




\begin{tabular}{|c|c|c|c|c|c|}
\hline \multicolumn{6}{|c|}{ Flower } \\
\hline 45 & 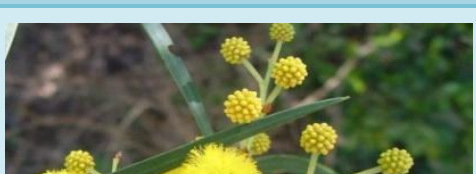 & $\mathrm{AQ}$ & $\begin{array}{l}\text { MIC, } \\
\text { DPPH }\end{array}$ & $\begin{array}{l}\text { Antifungal, } \\
\text { Antibacterial, } \\
\text { Antioxidant }\end{array}$ & $\begin{array}{l}\text { Al-Huqail et al., } \\
2019\end{array}$ \\
\hline & $\begin{array}{l}\text { Acacia saligna (Labill.) } \\
\text { H.L.Wendl./Fabaceae }\end{array}$ & & & & \\
\hline 46 & 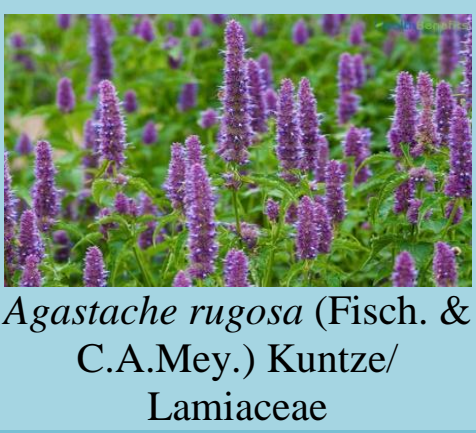 & $\begin{array}{l}\text { ET, } \\
\text { HE, } \\
\text { ME }\end{array}$ & $\begin{array}{c}\mathrm{DPPH}, \\
\mathrm{H}_{2} \mathrm{O}_{2}, \\
\mathrm{SO}, \\
\text { Agar disc } \\
\text { diffusion }\end{array}$ & $\begin{array}{l}\text { Antioxidant, } \\
\text { Antimicrobial }\end{array}$ & Park et al., 2019 \\
\hline 47 & $\begin{array}{c}\text { (c) } \\
\text { Aloe barbadensis (L.) } \\
\text { Burm.f./Asphodelaceae }\end{array}$ & $70 \%$ ET & $\begin{array}{l}\text { TPC, } \\
\text { TFC, } \\
\text { DPPH, } \\
\text { ABTS, } \\
\text { RP, } \\
\text { NO, } \\
\text { OH, } \\
\text { SO }\end{array}$ & Antioxidant & $\begin{array}{l}\text { Debnath et al., } \\
2018\end{array}$ \\
\hline 48 & & $\begin{array}{c}100 \% \mathrm{ME}, \\
80 \% \mathrm{AQ}\end{array}$ & TFC & $\begin{array}{l}\text { Hepato-protective, } \\
\text { Neuro-protective }\end{array}$ & $\begin{array}{l}\text { Kwon et al., } \\
2019\end{array}$ \\
\hline 49 & & $\begin{array}{l}\text { ET, } \\
\text { AQ }\end{array}$ & Phytochemical & $\begin{array}{l}\text { Acute toxicity, } \\
\text { Anti-ulcer }\end{array}$ & $\begin{array}{l}\text { Barakat et al., } \\
2019\end{array}$ \\
\hline & $\begin{array}{c}\text { Bombax ceiba L./ } \\
\text { Malvaceae }\end{array}$ & & & & \\
\hline
\end{tabular}




\begin{tabular}{|c|c|c|c|c|c|}
\hline 50 & $\begin{array}{c}\begin{array}{c}\text { Carica papaya } \mathrm{L} . / \\
\text { Caricaceae }\end{array} \\
\text { Caricas }\end{array}$ & $\begin{array}{l}\mathrm{ME}, \\
\mathrm{CF}, \\
\mathrm{n}-\mathrm{HE}, \\
\mathrm{AQ}\end{array}$ & $\begin{array}{c}\text { TPC, } \\
\text { TFC, } \\
\text { DPPH, } \\
\text { Agar well } \\
\text { diffusion }\end{array}$ & $\begin{array}{l}\text { Antioxidant, } \\
\text { Antibacterial }\end{array}$ & $\begin{array}{l}\text { Dwivedi et al., } \\
2020\end{array}$ \\
\hline 51 & Cassia fistula L./Fabaceae & - & - & $\begin{array}{c}\text { Anti- } \\
\text { inflammatory }\end{array}$ & $\begin{array}{l}\text { Antonisamy et } \\
\text { al., } 2019\end{array}$ \\
\hline 52 & $\begin{array}{c}\text { Ctenolepis garcini (L.) } \\
\text { C.B.Clarke/Cucurbitaceae }\end{array}$ & ET & AT & Antiulcer & $\begin{array}{l}\text { Kumar et al., } \\
2019\end{array}$ \\
\hline 53 & $\begin{array}{l}\text { Etlingera elatior (Jack) } \\
\text { R.M. Sm./Zingiberaceae }\end{array}$ & ET & $\begin{array}{l}\text { Agar disc } \\
\text { diffusion, } \\
\text { MIC, } \\
\text { MBC }\end{array}$ & Antibacterial & $\begin{array}{l}\text { Anzian et al., } \\
2020\end{array}$ \\
\hline 54 & $\begin{array}{c}\text { Pelago macrocarpa } \\
\text { Ferulago } \\
\text { (Fenzl) Boiss./Apiaceae }\end{array}$ & $\mathrm{AQ}$ & $\begin{array}{c}\text { (AgNPs) } \\
\text { DPPH, } \\
\text { RSA, } \\
\text { FTIR, } \\
\text { SEM, } \\
\text { EDX, } \\
\text { XRF, } \\
\text { XRD, } \\
\text { Agar disc } \\
\text { diffusion, } \\
\text { Agar well }\end{array}$ & $\begin{array}{l}\text { Antibacterial, } \\
\text { Anticancer, } \\
\text { Antifungal }\end{array}$ & $\begin{array}{l}\text { Azarbani and } \\
\text { Shiravand, } 2020\end{array}$ \\
\hline
\end{tabular}




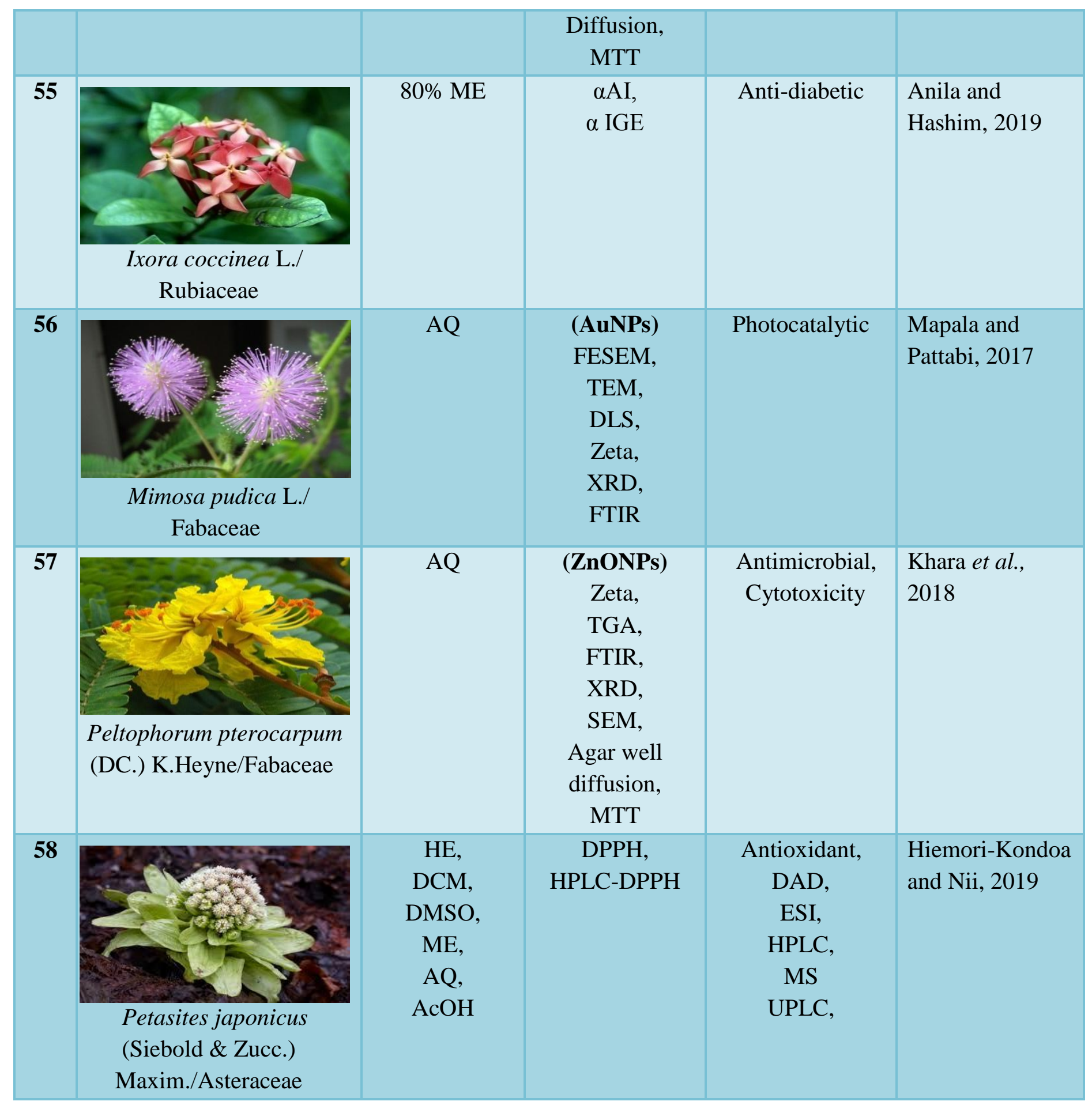




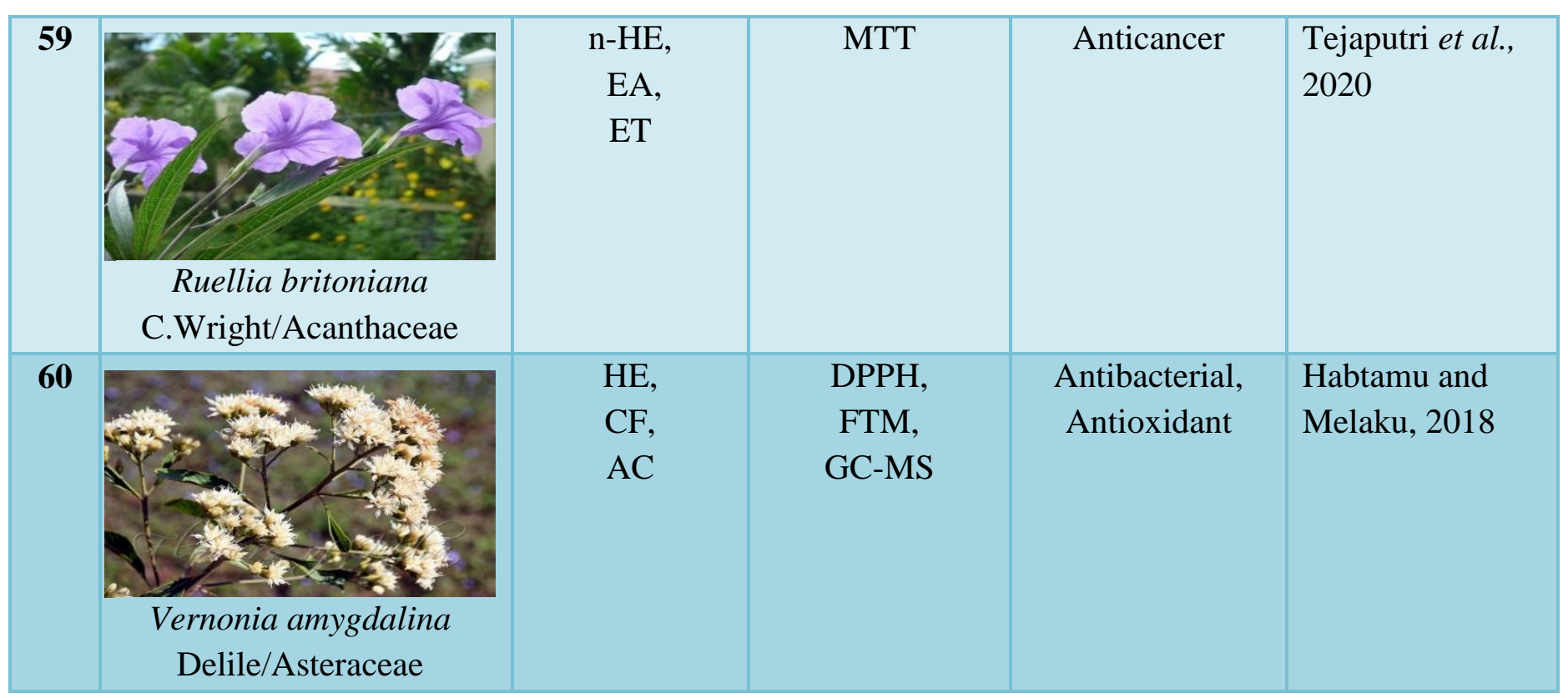

Begonia semperflorens flowers showed hepatoprotective and neuroprotective properties (Kwon et al., 2019). Ixora coccinea flowers were reported for antidiabetic property (Anila and Hashim, 2019). The presence of secondary metabolites like flavonoids, tannins and phenols in methanol extract were attributed for its antidiabetic property. Bombax ceiba flowers showed antiulcer property (Barakat et al., 2019). They contained phytoconstituents like flavonoids (naringenin), phenols (rutin, quercetin and gallic acid), carbohydrates, tannins, sterols, coumarins and other phytoconstituents like caffeic acid, ferulic acid, syringeic, cinnamic acid and mangiferin. Antiulcer activity was reported by flower extract of Ctenolepis garcini (Kumar et al., 2019); anticancer activity against HeLa cervical cancer cell line by Ruellia britoniana flowers (Tejaputri et al., 2020).

Like seeds and peels, flowers are also utilized for metal nanoparticle synthesis and they showed many biological activities. For e.g. silver nanoparticles synthesized using flower extract of Cassia roxburghii and Ferulago macrocarpa showed antibacterial and antifungal activities (Moteriya and Chanda, 2014; Azarbani and Shiravand, 2020). Titanium oxide nanoparticles synthesized using Hibiscus rosasinesis flower extract showed antibacterial activity (Kumar et al., 2014). Mimosa pudica flower extract mediated synthesized gold nanoparticles showed dye degradation or photocatalytic activity (Mapala and Pattabi, 2017). Peltophorum pterocarpum flower mediated synthesized zinc oxide nanoparticles showed antimicrobial and cytotoxic activities (Khara et al., 2018) while those from Nyctanthes arbor-tristis showed antifungal activity (Jamdagni et al., 2018). Copper oxide nanoparticles synthesized using Magnolia champaca floral extract showed antioxidant activity (Santhoshkumar and Shanmugam, 2020).

In this review, we report the biological activity of some seeds, peels and flowers. The Seeds belonged to 17 different families like Arecaceae, Asteraceae, Capparidaceae, Clusiaceae, Cucurbitaceae, Fabaceae, Lauraceae, Malvaceae, Moringaceae, Myrtaceae, Pedaliaceae, Rhamnaceae, Rosaceae, Rutaceae, Sapotaceae, Solanaceae 
and Zingiberaceae. The peels belonged to 15 families viz. Actinidiaceae, Amaryllidaceae, Anacardiaceae, Asphodelaceae, Bromeliaceae, Caricaceae, Cucurbitaceae, Lythraceae, Musaceae, Rhamnaceae, Rosaceae, Rutaceae, Sapindaceae, Sapotaceae and Solanaceae. The flowers belonged to 12 families viz. Acanthaceae, Apiaceae, Asphodelaceae, Asteraceae, Begoniaceae, Caricaceae, Cucurbitaceae, Fabaceae, Lamiaceae, Malvaceae, Rubiaceae and Zingiberaceae.

In conclusion, this review summarizes the therapeutic potential of biowaste of plants i.e. biological activities of seeds, peels and flowers. These parts which are generally thrown into the environment can be successfully exploited as a natural source for activities like antimicrobial, antioxidant, antidiabetic, anti-ulcer, hepatoprotective, anticancer, would healing, etc properties. These are mostly crude extracts therefore detailed studies have to be done using various extraction techniques and their mechanism of action has to be worked out. Structural characterization of the phytochemicals involved also needs to be worked out. But definitely they are not waste and can be therapeutically useful. It has double advantage of decreasing pollution and increasing naturally occurring bioactive compounds which can be therapeutically useful.

\section{Acknowledgements}

The authors thank Department of Biosciences (UGC-CAS) for providing excellent research facilities.

\section{Abbreviation}

AQ - Aqueous, ET - Ethanol, HE - Hexane, ME Methanol, CF - Chloroform, n-HE - n-Hexane, EA Ethyl acetate, AC - Acetone, PE - Petroleum ether, EtOH - Ethyl alcohol, DCM - Dichloro-methane,
DMSO - Di-methyl-sulfoxide, AcOH - Acetic acid, $\mathrm{CaSO}_{4}$ - Calcium sulfate, $\mathrm{CaCO}_{3}-$ Calcium carbonate, $\mathbf{C a C l}_{\mathbf{2}}$ - Calcium chloride, HME - Hydromethanolic solution, NHET - Hydro-ethanolic solution, PP - Propanone, TPC - Total phenolic, PAC - Phenolic acid content, TFC/FC - Total Flavonoids content, AC - Alkaloids content, TTP - Triterpenes, TT - Total tannins, TCP - Tocopherols phytosterols, TC - Total carotenoids, TTC - Tannins content, SC Saponins content, PPC - Polyphenol content, TMC Total mucilage content, DPPH - 2,2-diphenyl-1picrylhydrazyl, SO - Superoxide anion radical scavenging activity, ABTS - 2,2'-azino-bis (3ethylbenzothiazoline-6-sulfonic acid, FRAP - Ferric Reducing antioxidant Power, $\mathbf{O}_{2}-\mathrm{O}_{2}$ scavenging activity, $\mathbf{O H}$ - Hydroxyl free radical scavenging activity, $\mathbf{H}_{2} \mathbf{O}_{2}-$ Hydrogen peroxide activity, NO Nitric oxide-scavenging activity, TRP - Total reducing power, TAC - Total antioxidant capacity, RP Reducing power, RSA - Radical scavenging activity, TEAC - Trolox Equivalent Antioxidant Capacity, SAEA - Serum antioxidant enzyme activity, MIC Minimum inhibitory concentration, MBC - Minimum bactericidal concentration, MTP - Microtiter plate assay, MTT - 3-(4,5-dimethylthiazol-2-yl)-2,5diphenyl tetrazolium bromide, MCF-7 - Breast cancer cell line, HCT - Colon carcinoma cell line, BVA Bacterial viability assay, LDCA - Live and dead cell assay, HEPG2 - Liver carcinoma cell line, XRD - XRay Diffraction, XRF - X-ray fluorescence, Zeta Zeta potential, FTIR - Fourier Transform Infrared Spectroscopy, FESEM - Field Emission Scanning Electron Microscope, SEM - Scanning electron microscope, HR-TEM - High-resolution transmission electron microscopy, TEM - Transmission electron microscopy, SAED - Selected area diffraction, DLS Dynamic light scattering, EDX - Energy Dispersive XRay analysis, NMR - NMR spectrometric analysis, TG - Thermogravimetric analysis, GC-MS - Gas Chromatography Mass Spectrometry, HPLC - High Performance Liquid Chromatography, UPLC-MS Ultra performance liquid chromatography - mass spectrometer, NLA - Nitrite and Luciferase assay, PCA - Correlation analysis by principal component analysis, PSA - Phenol-sulfuric acid method, RAT Rhein acute toxicity, SLP - Serum lipid parameters, SPA - Spectro-photometric assay, SICA - Starchiodine colour assay, EPS - Exopolysaccharide quantification, FCC - Ferrous chelating capacity, FTM - Ferric tiocyanate method, $\boldsymbol{\alpha A I}-\alpha$-Amylase inhibition, BSL - Brine shrimp lethality, AT - Acute toxicity, HA - Hemolytic activity, HPA - Histopathological analysis, HIV - Human immunodeficiency virus, IHCA Immunohistochemical analysis, $\boldsymbol{\alpha}$ IGE - Inhibition of alpha-glucosidase enzyme, LAS - Linoleic acid 
system, LP - Lipid peroxidation, LTL - Liver total lipids, MDA - Serum malondialdehyde concentration, AAIE - Anti-acute inflammation effect, ASIE - Antisubacute inflammatory effect, CLC - Cell line culture, BSCA - Brine shrimp cytotoxicity assay, REMA REMA assay, AgNPs - Silver nanoparticles, AuNPs Gold nanoparticles, ZnONPs - Zinc oxide nanoparticles.

\section{References}

Abbasi BH, Shah M, Hashmi SS, Nazir M, Naz S, Ahmad W, Khan IU, Hano C (2019). Green bio-assisted synthesis, characterization and biological evaluation of biocompatible ZnONPs synthesized from different tissues of milk thistle (Silybum marianum). Nanomaterials. 9(8):1171. doi:10.3390/nano9081171

Abdel-Mageed WM, Bayoumi SAH, Chen C, Vavricka CJ, Li L, Malik AM, Dai H, Song F, Wang L, Zhang J, Gao GF, Lv Y, Liu L, Liu X, Sayad HM, Zhang L (2014). Benzophenone C-glucosides and gallotannins from mango tree stem bark with broad-spectrum anti-viral activity. Bioorganic \& Medicinal Chemistry. 22(7):2236-2243.

Abreu MM, Nobrega PD, Sales PF, FRDe O, Nascimento AA (2019). Antimicrobial and antidiarrheal activities of methanolic fruit peel extract of Pouteria caimito. Pharmacognosy Journal. 11(5): 944-950.

Abubakar A, Olorunkemi MR, Busari MB, Hamzah RU, Abdulrasheed-Adeleke T (2020). Comparative in vitro antioxidant activities of aqueous extracts of Garcinia kola and Buchholzia coriacea seeds. Tanzania Journal of Science. 46(2):498-507.

Agarwal R, Gupta R, Yadav R, Asati V, Rathi JC (2019). Anti-inflammatory activity of seeds extract of Datura stramonium against carrageenan induced paw edema on albino Wistar rats. Journal of Pharmaceutical and Biological Sciences. 7(1):41-46.

Al-Huqail AA, Behiry SI, Salem MZM, Ali HM, Siddiqui MH, Salem AZM (2019). Antifungal, antibacterial and antioxidant activities of Acacia saligna (Labill.) HL Wendl. flower extract: HPLC analysis of phenolic and flavonoid compounds.
Molecules.

$24: 700$ doi: 10.3390/molecules24040700.

Alim A, Li T, Nisar T, Ren D, Zhai X, Pang Y, Yang X (2019). Antioxidant, antimicrobial and antiproliferative activity-based comparative study of peel and flesh polyphenols from Actinidia chinensis. Food \& Nutrition Research. 63:1577. http://dx.doi. org/10. 29219/fnr.v63.1577.

Al-Nemari R, Bacha AB, Al-Senaidy A, Arafah M, Al-Saran H, Abutaha N, Semlali A (2020). Selective cytotoxic effects of Annona squamosa leaves against breast cancer cells via apoptotic signaling proteins. doi:10.20944/preprints202005.0212.v1.

Al-Zoreky N (2017). Antimicrobial activity of pomegranate (Punica granatum L.) fruit peels. International Journal of Food Microbiology. 134:244-248.

Amado DAV, Helmann GAB, Detoni AM, de Carvalho SLC, de Aguiar CM, Martin CA, Tiuman TS, Cottica SM (2019). Antioxidant and antibacterial activity and preliminary toxicity analysis of four varieties of avocado (Persea americana Mill.). Brazilian Journal of Food Technology. 22: https://doi.org/10.1590/1981-6723.04418.

Anila L and Hashim MS (2019). Evaluation of in vitro anti-diabetic activity of methanolic extract of Ixora coccinea flowers. Journal of Pharmaceutical Sciences and Research. 11(6):2367-2372.

Antonisamy P, Agastian P, Kang CW, Kim NS, Kim JH (2019). Anti-inflammatory activity of rhein isolated from the flowers of Cassia fistula L. and possible underlying mechanisms. Saudi Journal of Biological Sciences. 26(1):96-104.

Anzian A, Muhialdin BJ, Mohammed NK, Kadum H, Marzlan AA, Sukor R, Meor Hussin ASM (2020). Antibacterial activity and metabolomics profiling of torch ginger (Etlingera elatior Jack) flower oil extracted using subcritical carbon dioxide $\left(\mathrm{CO}_{2}\right)$. Evidence-Based Complementary and Alternative Medicine. https://doi.org/ 10.1155/2020/4373401.

Azarbani F and Shiravand S (2020). Green synthesis of silver nanoparticles by Ferulago macrocarpa flowers extract and their antibacterial, antifungal and toxic effects. 
Green Chemistry Letters and Reviews. 13(1):41-49.

Babatunde O, Oladeji RD, Ajayi IA (2019). Evaluation of wound healing potential of Chrysophyllum albidum hexane seed extract in Wistar rat. Journal of Biomedical Research and Clinical Practice. 2(1):82-90.

Bahramsoltani R, Farzaei MH, Abdolghaffari AH, Rahimi R, Samadi N, Heidari M, Esfandyari M, Baeeri M, Hassanzadeh G, Abdollahi M, Soltani S, Pourvaziri A, Amin G (2017). Evaluation of phytochemicals, antioxidant and burn wound healing activities of Cucurbita moschata Duchesne fruit peel. Iranian Journal of Basic Medical Sciences. 20(7):798-805.

Barakat MMA, El-Boghdady NA, Farrag EKE, Said AA, Shaker SE (2019). Protective and curative effects of Bombax ceiba flower and Ziziphus spina Christi fruit extracts on gastric ulcer. Journal of Biological Sciences. 19(2):161-172.

Baravalia Y and Chanda S (2011). Protective effect of Woodfordia fruticoza Kurz. flowers against acetaminophen induced hepatic toxicity in rats. Pharmaceutical Biology. 49(8):826-832.

Barbi RC, Teixeira GL, Hornung PS, Avila S, Ribani RH (2018). Eriobotrya japonica seed as a new source of starch: Assessment of phenolic compounds, antioxidant activity, thermal, rheological and morphological properties. Food Hydrocolloids. 77:646-658.

Basri HH, Talib RA, Sukor R, Othman SH, Ariffin H (2020). Effect of synthesis temperature on the size of $\mathrm{ZnO}$ nanoparticles derived from pineapple peel extract and antibacterial activity of $\mathrm{ZnO}-$ Starch nanocomposite films. Nanomaterials. 10(6):1061. doi:10.3390/nano10061061.

Chanda S and Nagani K (2013). In vitro and in vivo methods for anticancer activity evaluation and some Indian medicinal plants possessing anticancer properties: An overview. Journal of Pharmacognosy and Phytochemistry. 2(2): 140-152.

Chanda S, Rakholiya K, Parekh J (2013). Indian medicinal herb: Antimicrobial efficacy of Mesua ferrea L. seed extracted in different solvents against infection causing pathogenic strains. Journal of Acute Diseases. 2(4): 277-
281.

Christijanti W, Juniarto AZ, Suromo LB (2019). Aloe Vera peel extract administration increased antioxidant enzyme levels of serum and seminal plasma in type 2 diabetic rats. Pharmacognosy Journal. 11(5):962-967.

Chueh CC, Lin LJ, Lin WC, Huang SH, Jan MS, Chang SC, Chung WS, Lee TT (2019). Antioxidant capacity of banana peel and its modulation of Nrf2-ARE associated gene expression in broiler chickens. Italian Journal of Animal Science. 18(1):13941403.

Debnath T, Ghosh M, Lee YM, Nath NCD, Lee KG, Lim BO (2018). Identification of phenolic constituents and antioxidant activity of Aloe barbadensis flower extracts. Food and Agricultural Immunology. 29(1):27-38.

Donga S, Pande J, Moteriya P, Chanda S (2017a). In vitro cytotoxicity study of leaf, stem and bark of Pterocarpus santalinus Linn. F. Journal of Pharmacognosy and Phytochemistry. 6(4):297-305.

Donga S, Moteriya P, Chanda S (2017b). Evaluation of antimicrobial and synergistic antimicrobial properties of Pterocarpus santalinus. Asian Journal of Pharmaceutical and Clinical Research. 10(11):204-209.

Dwivedi MK, Sonter S, Mishra S, Patel DK, Singh PK (2020). Antioxidant, antibacterial activity and phytochemical characterization of Carica papaya flowers. Beni-Suef University Journal of Basic and Applied Sciences. $9(23)$ : https://doi.org/10.1186/s43088-020-00048w.

Eggadi V, Lingampalli J, Kamma S, Bandaru SBS, Alavala RR, Kulandaivelu U (2019). Evaluation of antiulcer activity of Lawsonia inermis and Murraya koenigii seed extract in ethanol-induced gastric mucosal damage in rats. Asian Journal of Biological Sciences. 12(4): 884-890.

El-Mesallamy AMD, El-Latif AESA, El-Azim MHA, Mahdi MGM, Hussein SAM (2020). Chemical composition and biological activities of red beetroot (Beta Vulgaris Linnaeus) roots. Egyptian Journal of Chemistry. 63(1): 239-246.

El-Toumy SAH, Salib JY, Shafik NH, Elkarim 
ASA, Salama A, Omara EAA, Micky J (2019). Evaluation of hepatoprotective activity of Polygonum equisetiforme methanolic extract. Journal of Applied Pharmaceutical Science. 9(11):54-59.

Fidelis M, Santos JS, Escher GB, do Carmo MV, Azevedo L, da Silva MC, Putnik P, Granato $\mathrm{D}$ (2018). In vitro antioxidant and antihypertensive compounds from camucamu (Myrciaria dubia McVaugh, Myrtaceae) seed coat: A multivariate structure-activity study. Food and Chemical Toxicology. 120:479-490.

Ghasemzadeh A, Jaafar HZ, Bukhori MF, Rahmat MH, Rahmat A (2018). Assessment and comparison of phytochemical constituents and biological activities of bitter bean (Parkia speciosa Hassk.) collected from different locations in Malaysia. Chemistry Central Journal. 12(12): https://doi.org/10.1186/s13065-018-0377-6.

Golechha M, Sarangal V, Ojha S, Bhatia J, Arya DS (2014). Anti-inflammatory effect of Emblica officinalis in rodent models of acute and chronic inflammation: involvement of possible mechanisms. International Journal of Inflammation. http://dx.doi.org/10. 1155/2014/178408.

Habtamu A and Melaku Y (2018). Antibacterial and antioxidant compounds from the flower extracts of Vernonia amygdalina. Advances in Pharmacological Sciences. https://doi.org/10.1155/2018/4083736.

Hiemori-Kondo M and Nii M (2019). In vitro and in vivo evaluation of antioxidant activity of Petasites japonicus Maxim. flower buds extracts. Bioscience, Biotechnology and Biochemistry. 84(3):621-632.

Huang CY, Kuo CH, Wu CH, Kuan AW, Guo HR, Lin YH, Wang PK (2018). Free radicalscavenging, anti-inflammatory and antibacterial activities of water and ethanol extracts prepared from compressionalpuffing pretreated mango (Mangifera indica L.) peels. Journal of Food Quality. https://doi.org/10.1155/2018/1025387.

Iman S, Saleem U, Ahmad B (2020). Preclinical assessment of antiurolithiatic activity of Mangifera indica seeds on ethylene glycol induced urolithiasis rat model. Pakistan Veterinary Journal. doi: 10.29261/pakvetj/2020.028.

Ishwarya R, Vaseeharan B, Anuradha R, Rekha R, Govindarajan M, Alharbi NS, Kadaikunnan S, Khaled JM, Benelli G (2017). Ecofriendly fabrication of $\mathrm{Ag}$ nanostructures using the seed extract of Pedalium murex, an ancient Indian medicinal plant: Histopathological effects on the Zika virus vector Aedes aegypti and inhibition of biofilm-forming pathogenic bacteria. Journal of Photochemistry \& Photobiology, B: Biology. 174:133-143.

Islam R and Sultana B (2020). Investigation of the antioxidant activity in melon peel and seeds (Cucumis melo). Journal of Applied Sciences and Research. 1(1):1-6.

Jamdagni P, Khatri P, Rana JS (2018). Green synthesis of zinc oxide nanoparticles using flower extract of Nyctanthes arbor-tristis and their antifungal activity. Journal of King Saud University - Science. doi:10.1016/j.jksus.2016.10.002.

Jorge N, Silva AC, Aranha CP (2016). Antioxidant activity of oils extracted from orange (Citrus sinensis) seeds. Anais da Academia Brasileira de Ciencias. 88(2):951958.

Kanbargi KD, Sonawane SK, Arya SS (2016). Functional and antioxidant activity of Ziziphus jujube seed protein hydrolysates. Journal of Food Measurement and Characterization. 10(2):226-235.

Kaneria MJ, Bapodara MB, Chanda SV (2012). Effect of extraction techniques and solvents on antioxidant activity of pomegranate (Punica granatum L.) leaf and stem. Food Analytical Methods. 5(3):396-404.

Khara G, Padalia H, Moteriya P, Chanda S (2018). Peltophorum pterocarpum flowermediated synthesis, characterization, antimicrobial and cytotoxic activities of Zno nanoparticles. Arabian Journal for Science and Engineering. 43(7):3393-3401.

Khemiri I and Bitri L (2019). Effectiveness of Opuntia ficus indica $\mathrm{L}$. inermis seed oil in the protection and the healing of experimentally induced gastric mucosa ulcer. Oxidative Medicine and Cellular Longevity. https://doi.org/10.1155/2019/1568720.

Kumar CP, Prathap P, Kazmi MH, Darshanala A 
(2019). Evaluation of antiulcer activity by using flower extract of Ctenolepis garcini in aspirin induced rats. International Journal of Research and Review. 6(11):209-213.

Kumar PS, Durgadevi S, Saravanan A, Uma S (2019). Antioxidant potential and antitumour activities of Nendran banana peels in breast cancer cell line. Indian Journal of Pharmaceutical Sciences. 81(3):464-473.

Kumar PSM, Francis AP, Devasena T (2014). Comparative studies on green synthesized and chemically synthesized titanium oxide nanoparticles. A validation for green synthesis protocol using Hibiscus flower. Journal of Environmental Nanotechnology. 3(4):78-85.

Kwon JH, Oh HJ, Lee DS, In SJ, Seo KH, Jung JW, Cha BJ, Lee DY, Baek NI (2019). Pharmacological activity and quantitative analysis of flavonoids isolated from the flowers of Begonia semperflorens Link et Otto. Applied Biological Chemistry. 62(1): https://doi.org/10.1186/s13765-019-0416-6.

Lolok N, Mashar HM, Annah I, Saleh A, Yuliastri WO, Isrul M (2019). Antidiabetic effect of the combination of garlic peel extract (Allium sativum) and onion peel (Allium сера) in rats with oral-glucose tolerance method. Research Journal of Pharmacy and Technology. 12(5):2153-2156.

Ma J-Shuang, Liu H, Han CR, Zeng SJ, Xu XJ, $\mathrm{Lu}$ DJ, He HJ (2019). Extraction, characterization and antioxidant activity of polysaccharide from Pouteria campechiana seeds. Carbohydrate Polymers. doi: https://doi.org/10.1016/ j.carbpol. 2019.115409.

Ma Q, Guo Y, Sun L, Zhuang Y (2017). Antidiabetic effects of phenolic extract from rambutan peels (Nephelium lappaceum) in high-fat diet and streptozotocin-induced diabetic mice. Nutrients. 9(8):801. doi:10.3390/nu9080801.

Mapala K and Pattabi M (2017). Mimosa pudica flower extract mediated green synthesis of gold nanoparticles. Nano World Journal. $3(2): 44-50$.

Mastrogiovanni F, Mukhopadhya A, Lacetera N, Ryan MT, Romani A, Bernini R, Sweeney T (2019). Anti-inflammatory effects of pomegranate peel extracts on in vitro human intestinal caco-2 cells and ex vivo porcine colonic tissue explants. Nutrients. 11(3):548. doi:10.3390/nu11030548.

Metoui M, Essid A, Bouzoumita A, Ferchichi A (2019). Chemical composition, antioxidant and antibacterial activity of Tunisian date palm seed. Polish Journal of Environmental Studies. 28(1):267-274.

Mokbel MS and Hashinaga F (2005). Antibacterial and antioxidant activities of banana (Musa, AAA cv. Cavendish) fruits peel. American Journal of Biochemistry and Biotechnology. 1(3):125-131.

Moopayak W and Tangboriboon N (2020). Mangosteen peel and seed as antimicrobial and drug delivery in rubber products. Journal of Applied Polymer Science. https://doi.org/ 10.1002/app.49119.

Moteriya P and Chanda S (2014). Biosynthesis of silver nanoparticles using flower extract of Cassia roxburghii DC. and its synergistic antibacterial efficacy. Scientia Iranica. Transaction F: Nanotechnology. 21(6):24992507.

Motohashi N, Shirataki Y, Kawase M, Tani S, Sakagami H, Satoh K, Kurihara T, Nakashima H, Wolfard K, Miskolci C, Molnar J (2001). Biological activity of kiwifruit peel extracts. Phytotherapy Research. 15(4):337-343.

Osarumwense PO (2017). Anti-inflammatory activity of methanoilc and ethanolic extracts of Citrus sinensis peel (L.) Osbeck on carrageenan induced paw oedema in Wistar rats. Journal of Applied Sciences and Environmental Management. 21(6):12231225.

Pande J and Chanda S (2020). Determination of phytochemical profile and antioxidant efficacy of Lavendula bipinnata leaves collected during Magha Nakshatra days and Normal days using LC-QTOF-MS technique. Journal of Pharmaceutical and Biomedical Analysis. https://doi.org/10.1016/j.jpba.2020.113347.

Parikh B and Patel VH (2017). Quantification of phenolic compounds and antioxidant capacity of an underutilized Indian fruit: Rayan [Manilkara hexandra (Roxb.) Dubard]. Food Science and Human Wellness. 6(1):10-19. 
Park CH, Yeo HJ, Baskar TB, Park YE, Park JS, Lee SY, Park SU (2019). In vitro antioxidant and antimicrobial properties of flower, leaf, and stem extracts of Korean mint. Antioxidants. $8: 75$. doi:10.3390/antiox8030075.

Paseban M, Niazmand S, Soukhtanloo M, Meybodi NT (2020). The preventive effect of Nigella sativa seed on gastric ulcer induced by indomethacin in rat. Journal of Herbmed Pharmacology. 9(1):12-19.

Patra JK and Baek KH (2015). Novel green synthesis of gold nanoparticles using Citrullus lanatus rind and investigation of proteasome inhibitory activity, antibacterial and antioxidant potential. International Journal of Nanomedicine. 10:7253-7264.

Patra JK and Baek KH (2016). Green synthesis of silver chloride nanoparticles using Prunus persica L. outer peel extract and investigation of antibacterial, anticandidal, antioxidant potential. Green Chemistry Letters and Reviews. 9(2):132-142.

Queiroz ER, de Abreu CMP, Rocha DA, de Sousa RV, Fraguas RM, Braga MA, Cesar PHS (2018). Lychee (Litchi chinensis Sonn.) peel flour: effects on hepatoprotection and dyslipidemia induced by a hypercholesterolemic diet. Anais da Academia Brasileira de Ciencias. 90(1):267281.

Rajan A, Rajan AR, Philip D (2017). Elettaria cardamomum seed mediated rapid synthesis of gold nanoparticles and its biological activities. Open Nano. 2:1-8.

Rakholiya K, Kaneria M, Chanda S (2014). Inhibition of microbial pathogens using fruit and vegetable peel extracts. International Journal of Food Science and Nutrition. 65(6): 733-739.

Rakholiya KD, Kaneria MJ, Chanda S (2015) In vitro assessment of novel antimicrobial from methanol extracts of matured seed kernel and leaf of Mangifera indica L. (Kesar mango) for inhibition of Pseudomonas spp. and their synergistic potential. American Journal of Drug Discovery and Development. 5(1):13-23.

Rebello LPG, Ramos AM, Pertuzatti PB, Barcia MT, Castillo-Munoz N, Hermosin-Gutierrez I (2014). Flour of banana (Musa AAA) peel as a source of antioxidant phenolic compounds. Food Research International. 55:397-403.

Rochin-Medina JJ, Sotelo-Castro JA, SalazarSalas NY, Lopez-Valenzuela JA, Ramirez K (2019). Antioxidant and anti-Salmonella activities of eggplant peel compounds obtained by solvent-free calcium-based extraction. CyTA-Journal of Food. 17(1):873-881.

Rodriguez-Carpena J, Morcuende D, Andrade MJ, Kylli P, Estevez M (2011). Avocado (Persea americana Mill) phenolics, in vitro antioxidant and antimicrobial activities, and inhibition of lipid and protein oxidation in porcine patties. Journal of Agricultural and Food Chemistry. 59(10):5625-5635.

Rolim PM, Fidelis GP, Padilha CE, Santos ES, Rocha HA, Macedo GR (2018). Phenolic profile and antioxidant activity from peels and seeds of melon (Cucumis melo L. var. reticulatus) and their antiproliferative effect in cancer cells. Brazilian Journal of Medical and Biological Research. 51(4): doi.org/10.1590/1414-431x20176069.

Saleem M and Saeed MT (2020). Potential application of waste fruit peels (orange, yellow lemon and banana) as wide range natural antimicrobial agent. Journal of King Saud University-Science. 32(1):805-810.

Santhoshkumar J and Shanmugam VK (2020). Green synthesis of copper oxide nanoparticles from magnolia champaca floral extract and its antioxidant \& toxicity assay using danio rerio. International Journal of Recent Technology and Engineering. 8(5):5444-5449.

Selim YA, Mohamed H, Hussien SY (2019). Efficacious anti-cancer property of liver from peels extract of Egyptian Citrus reticulate. Journal of Nutrition and Food Sciences. 7(3): id1118.

Siddique S, Nawaz S, Muhammad F, Akhtar B, Aslam B (2018). Phytochemical screening and in-vitro evaluation of pharmacological activities of peels of Musa sapientum and Carica papaya fruit. Natural Product Research. 32(11):1333-1336.

Singh AK, Singh S, Chandel HS (2012). Evaluation of hepatoprotective activity of Abelmoschus moschatus seed in paracetamol 
induced hepatotoxicity on rat. IOSR Journal of Pharmacy. 2(5):43-50.

Sukri SNAM, Shameli K, Wong MMT, Teow SY, Chew J, Ismail NA (2019). Cytotoxicity and antibacterial activities of plant-mediated synthesized zinc oxide $(\mathrm{ZnO})$ nanoparticles using Punica granatum (pomegranate) fruit peels extract. Journal of Molecular Structure. 1189:57-65.

Samuggam S, Vasanthi S, Shalini S, Chinni SV, Gopinath SCB, Anbu P, Bahari MB, Harish R, Kathiresan S, Ravichandran V (2018). Phyto-mediated photo catalysed green synthesis of silver nanoparticles using Durio zibethinus seed extract: antimicrobial and cytotoxic activity and photocatalytic applications. Molecules. 23(12):3311. doi:10.3390/ molecules 23123311.

Tejaputri NA, Arsianti A, Qorina F, Fithrotunnisa Q, Azizah NN, Putrianingsih R (2020). Anticancer activity of Ruellia britoniana flower on cervical HeLa cancer cells. Pharmacognosy Journal. 12(1):29-34.

Varghese R, Almalki MA, Ilavenil S, Rebecca J, Choi KC (2019). Silver nanopaticles synthesized using the seed extract of Trigonella foenum-graecum L. and their antimicrobial mechanism and anticancer properties. Saudi Journal of Biological Sciences. 26:148-154.
Vu HT, Scarlett CJ, Vuong QV (2018). Phenolic compounds within banana peel and their potential uses: A review. Journal of Functional Foods. 40:238-248.

Wadhwa M, Bakshi MPS, Makkar HPS (2015). Wastes to worth: value added products from fruit and vegetable wastes. CAB Reviews. 43: doi: 10.1079/PAVSNNR201510043.

Wang B, Hui Y, Liu L, Zhao A, Chiou YS, Zhang F, Pan MH (2019). Optimized extraction of phenolics from jujube peel and their antiinflammatory effects in LPS-stimulated murine macrophages. Journal of Agricultural and Food Chemistry. 67(6):1666-1673.

Xu YB, Chen GL, Guo MQ (2019). Antioxidant and anti-inflammatory activities of the crude extracts of Moringa oleifera from Kenya and their correlations with flavonoids. Antioxidants. 8(8):296. doi:10.3390/antiox8080296.

Yemis GP, Bach S, Delaquis P (2019). Antibacterial activity of polyphenol-rich pomegranate peel extract against Cronobacter sakazakii. International Journal of Food Properties. 22(1):985-993.

Zhao YL, Yang XW, Wu BF, Shang JH, Liu YP, Zhi-Dai, Luo XD (2019). Anti-inflammatory effect of pomelo peel and its bioactive coumarins. Journal of Agricultural and Food Chemistry. 67(32):8810-8818.

\section{How to cite this article:}

Savan Donga and Sumitra Chanda. 2020. Best from Waste: Therapeutic Potential of Plant Waste (Seeds, Peels, Flowers). Int.J.Curr.Microbiol.App.Sci. 9(08): 2670-2696.

doi: https://doi.org/10.20546/ijcmas.2020.908.305 